\title{
Protection evaluation against Chlamydophila abortus challenge by DNA vaccination with a dnaK-encoding plasmid in pregnant and non-pregnant mice
}

\author{
Céline HÉCHARD, Olivier GRÉPINET*, Annie RODOLAKIS
}

Unité de Pathologie Infectieuse et Immunologie, INRA, Centre de Tours, 37380 Nouzilly, France

(Received 23 November 2001; accepted 31 January 2002)

\begin{abstract}
Mice were intramuscularly immunized with a $d n a K$-encoding DNA plasmid. The protective effect of DNA immunization against Chlamydophila abortus infection was studied in pregnant and non-pregnant mice models. In non-pregnant mice, the $d n a K$ vaccine induced a specific humoral response with the predominant $\operatorname{IgG} 2 \mathrm{a}$ isotype, which failed to have in vitro neutralizing properties. No delayed-type hypersensitivity reaction was observed and the spleens of $d n a K$ vaccinated-mice were not protected against $C$. abortus challenge. In pregnant mice, the dnaK vaccine induced a non-specific partial protection from abortion. This may be due to the immunogenic properties of the CpG motifs of bacterial DNA present in the vaccinal plasmid backbone. Nevertheless, spleens of dnaK vaccinated-pregnant mice were not protected.
\end{abstract}

DNA immunization / Chlamydophila abortus / DnaK / Hsp70

Résumé - Évaluation de la protection induite par vaccination ADN avec un plasmide codant pour dnaK contre une épreuve par Chlamydophila abortus chez des souris gestantes ou nongestantes. Des souris ont été immunisées par l'injection intramusculaire d'un plasmide codant pour la protéine DnaK. L'effet protecteur de la vaccination ADN contre une infection par Chlamydophila abortus a été évalué dans des modèles de souris gestantes ou non-gestantes. La vaccination ADN avec le gène dnaK induit une réponse humorale spécifique chez les souris non-gestantes avec une prédominance d'IgG2a qui n'ont pas présenté d'activité neutralisante in vitro. Aucune réaction d'hypersensibilité retardée n'a été observée et les rates des souris vaccinées avec le vaccin ADN-dnaKn'ont pas été protégées contre l'infection par C.abortus. Chez les souris gestantes, le vaccin ADN-dnaK réduit partiellement et de façon non spécifique les avortements. Les îlots CpG de l'ADN bactérien, connus pour leur propriétés immunogéniques, pourraient être responsables de cet effet protecteur non spécifique. Cependant, aucune élimination des bactéries n'a été observée dans les rates des souris gestantes.

vaccination ADN / Chlamydophila abortus / DnaK / Hsp70

*Correspondence and reprints

Tel.: (33) 2474278 63; fax: (33) 2474277 79; e-mail: grepinet@tours.inra.fr 


\section{INTRODUCTION}

Chlamydophila abortus (Chlamydia psittaci serotype 1) is a Gram-negative obligate intracellular bacterial pathogen colonizing epithelial cells at mucosal surfaces. They notably infect cells of the reproductive tract and then are responsible for abortion of goats and sheep [18]. This pathology causes a considerable loss in breeding. Furthermore, it presents a zoonotic risk to pregnant women, since several cases of human chlamydial abortion have been reported [5]. A live attenuated vaccine 1B [24] is actually commercialized for small ruminants. It induces a specific and strong immunity that protects against infection but unfortunately does not enable the serological distinction between vaccinated and naturally infected animals [25].

DNA vaccination represents an exciting means to compensate for this $1 \mathrm{~B}$ vaccine disadvantage in inducing an immune response against a single $C$. abortus specific protein. In fact, it consists of direct gene transfer into the muscle or skin of a purified DNA plasmid encoding a gene of an immunogenic protein [13]. Its expression in the host cells results in the presentation of the protein to the immune system and leads to strong and persistent humoral and cell-mediated immune responses particularly appropriate for preventing intracellular bacterial infection [29]. Moreover, DNA vaccination generates the same Th1-biased immune response [31] implicated in the resolution of chlamydial infection and can be relevant to chlamydial vaccine design [6].

A large range of protein can be used in this kind of strategy. Proteins of the heat shock protein (Hsp) family are evaluated as good candidates for this approach of vaccination against $C$. abortus [1]. Widely distributed in nature, they are implicated in the folding, unfolding and translocation of proteins and also in the assembly and disassembly of protein complexes as immuno- globulins (Ig), T-cell receptors (TCR) and major histocompatibility complexes (MHC) [34]. Moreover, Hsp represent immunodominant targets inducing strong humoral and cellular immune responses [28] which are very interesting because they are similar to the one obtained after Chlamydiacae infection. Finally Hsp proteins held our attention because they have been successfully used in DNA vaccination of other models. Despite the potential immunopathological effect of Hsp, DNA vaccination with a dnaK gene encoding the DnaK protein which belongs to the Hsp family, offers an effective protection against tuberculosis without inducing adverse effects [16]. The DnaK protein $(70 \mathrm{kDa})$ is expressed in the early stages of the Chlamydia trachomatis infection [17] and presents several epitopes among which two are conformational [4] and one is linear [3].

In this study, we isolated the dnaK gene from a C. abortus AB7 genomic library and cloned it into a eukaryotic expression vector. Then we evaluated the protective effect in a pregnant and non-pregnant mice model of DNA immunization with the dnaK gene. The humoral immune response was examined by measuring isotype-specific antiDnaK antibodies.

\section{MATERIALS AND METHODS}

\subsection{C. abortus strains}

The virulent $C$. abortus $\mathrm{AB} 7$ ovine abortion strain isolated from an ovine abortion [14] and the vaccinal 1B strain obtained by selection of a temperature sensitive mutant after nitrosoguanidine mutagenesis [22] were used. Bacteria were propagated in the yolk sac of embryonated SPF chicken eggs inoculated at day 7 , purified as previously described [7] and stored at $-70{ }^{\circ} \mathrm{C}$. 


\subsection{Molecular cloning and expression of $C$. abortus dnaK gene}

\subsubsection{C. abortus $A B 7$ genomic library}

After lysis of $C$. abortus bacteria by treatment with proteinase $\mathrm{K}(2 \mathrm{mg} / \mathrm{mL})$ and L-laurylsarcosine (2\%), genomic DNA of C. abortus was extracted as previously described [12]. The genomic library of $C$. abortus DNA was constructed with the LambdaGEM $\AA 12$ kit (Promega Corporation, Madison, WI, USA) and according to the recommendations of the manufacturer. Briefly, after a partial digestion by Sau3A and a partial fill-in, DNA genomic fragments of C. abortus were cloned into XhoI digested lambda vector arms. This ligated DNA was packaged and used for infecting Escherichia coli KW251. After the phage transfer to a nitrocellulose filter (Schleicher $\&$ Schuell $0.45 \mu \mathrm{m}, \varnothing 82 \mathrm{~mm}$ ), the library was screened with the anti-DnaK monoclonal (Mab) 18.1 antibody [4] kindly provided by Dr S. Birkelund (University of Aarhus, Denmark).

Different cycles of purification and enrichment were done and we obtained a clone expressing the DnaK protein. The phagic DNA was extracted and in order to obtain the sequence of the dnaK gene, the corresponding insert was cloned into pBluescript ${ }^{\circledR}$ II $\mathrm{KS}+$ plasmid after a BamHI-SacI double digestion. The plasmid of the positive clones was sequenced (Genome Express, Meylan, France). The nucleotide sequence of the dnaK operon was then deposited in GenBank under the AF384685 accession number.

\subsubsection{Plasmid construction}

In order to produce the recombinant DnaK protein and vaccinal vector, the $d n a K$ gene was amplified by the polymerase chain reaction (PCR) and cloned in the appropriate vectors. PCR was performed using chlamydial genomic DNA (80 ng) as the template, dNTP $(200 \mu \mathrm{M}$ each $)$, specific primers ( $1 \mu \mathrm{M}$ each) and 1 unit of Taq DNA polymerase (Promega) in a Perkin Elmer thermocycler with the following program: 1 cycle of $94^{\circ} \mathrm{C} / 5 \mathrm{~min} ; 30$ cycles of $50{ }^{\circ} \mathrm{C} / 45 \mathrm{~s}, 72{ }^{\circ} \mathrm{C} / 2 \mathrm{~min}$ and $94^{\circ} \mathrm{C} / 45 \mathrm{~s}$; and 1 cycle of $50^{\circ} \mathrm{C} / 45 \mathrm{~s}, 72^{\circ} \mathrm{C} / 10 \mathrm{~min}$. Specific primers were determined according to the DNA sequence of C. abortus AB7 containing: (i) SacI site in the forward primer (DNAK-HIS1: 5'>: CTC GAG CTC AGC GAA CAA AAA AAA TCT AGT 3') and $K p n I$ site in the reverse primer (DNAK-HIS2: 5'>: CTC GGT ACC TTA ATC GTT AGG TTT ATC TAC 3'). The resulting fragment was inserted in a 5 '-end histidine tag $\left(\mathrm{His}_{6}\right)$ prokaryotic expression vector, pTrcHisA (Invitrogen, San Diego, CA, USA) linearized by KpnI-SacI double digestion to generate pTrcHis::DnaK; (ii) BamHI site and Kozak sequence in the forward primer (DNAK-B: 5'>: CTC GGA TTC ACC ATG AGC GAA CAA AAA AAA 3') and $X b a \mathrm{I}$ site in the reverse primer (DNAK-X: $5^{\prime}>$ : CTC TCT AGA TTA ATC GTT AGG TTT ATC TAC 3'). The resulting fragment was inserted into the pcDNA3.1 (Invitrogen) eukaryotic vaccinal vector carrying the human cytomegalovirus immediate early-promoter and the bovine growth hormone polyadenylation signal after linearization by BamHI-XbaI double digestion to generate pcDNA3.1::DnaK. pcDNA3.1::DnaK and pcDNA3.1 control plasmid were purified after an overnight Luria Bertani (LB) culture of recombinant $\mathrm{DH} 5 \alpha E$. coli $\mathrm{using}$ the EndoFree ${ }^{\mathrm{TM}}$ Plasmid Mega Kit (Qiagen, Hilden, Germany). Plasmid concentrations were determined by optical density, gel quantification and fitted to $1 \mathrm{mg} / \mathrm{mL}$ in PBS endofree. The sequence of the recombinant DnaK DNA was verified by DNA sequencing.

\subsubsection{Recombinant DnaK}

E. coli TG1 were transformed by pTrcHis::DnaK plasmid and grown overnight in $3 \mathrm{~mL}$ of LB supplemented with ampicillin $(100 \mu \mathrm{g} / \mathrm{mL})$. One milliliter of 
this culture was transferred into $100 \mathrm{~mL}$ of an LB-ampicillin medium. After $3 \mathrm{~h}$ of growth, isopropyl- $\beta$-D-thiogalactopyranoside (IPTG) was added at a final concentration of $1 \mathrm{mM}$ and then the culture was let to grow overnight. Bacteria were pelleted by centrifugation for $15 \mathrm{~min}$ at $3000 \mathrm{rpm}$. Then the recombinant DnaK protein was extracted according to the protocol of the Xpress ${ }^{\mathrm{TM}}$ System Protein Purification (Invitrogen), column washing and elution were performed under native conditions, then proteins were concentrated using an Ultrafree-4 centrifugal filter (Millipore, Bedford, MA, USA). Concentration of recombinant DnaK was evaluated by the BCA Protein Assay Reagent (Pierce, Rockford, IL, USA) and fitted to $1 \mathrm{mg} / \mathrm{mL}$ in PBS buffer.

\subsubsection{Eukaryotic expression of recombinant DnaK}

COS-7 cells were cultivated in DMEM-SVF $10 \%$ and transfected with Lipofectamine (Life Technologies Inc., Gaithersburg, MD, USA) by $1 \mu \mathrm{g}$ of plasmid per 3 to $4 \times 10^{5}$ cells. The expression of recombinant DnaK in the cell extract was tested after SDS-gel electrophoresis and western blot (see below) revelation with the anti-DnaK specific monoclonal 18.1 antibody and by immunofluorescence.

\subsection{Immunization and challenge}

All the studies were done on six-weekold female outbred OF1 Swiss mice (IFFA Credo, L'Arbresle, France).

Five groups of 20 mice for abortion tests were made. Prior to DNA immunization, each mouse was injected with cardiotoxin (Latoxan, Rosans, France) into the tibialis anterior muscles of both hind legs. Cardiotoxin induces a local inflammation and enhances the uptake of plasmid DNA [9]. Five days later, the mice were anesthetized by intraperitoneal injection of ketamine and xylazine ( 80 and $8 \mathrm{mg} / \mathrm{kg}$ of body weight, respectively) and immunized with pcDNA3.1 or pcDNA3.1::DnaK plasmids by intramuscular injections $(50 \mu \mathrm{g}$ in each tibialis anterior). The mice were boosted in the same way at days 21 and 42, mated at day 51 and challenged at day 63 by an intraperitoneal injection of $2 \times 10^{5}$ plaque forming units (pfu) of C. abortus AB7.

Additional control groups were done. First, a group of vaccinated control mice was obtained after one injection of $10^{5} 1 \mathrm{~B}$ vaccine bacteria at day 1 . The mice belonging to the virulence control group were infected but not immunized. Finally, mice of the pregnancy group were neither immunized nor challenged. Mice were considered protected when the number of living offspring per litter 8 days after birth was significantly different $(p<0.05)$ from the number of the control group as previously described [22].

Four groups of 16 non-pregnant mice were made for immunological trials. Non-pregnant mice were used in order to collect samples without affecting the pregnancy of the mice. Moreover, a good vaccine must be able to protect pregnant and non-pregnant animals. As for the mice of the abortion test, these non-pregnant mice were immunized with the $1 \mathrm{~B}$ vaccine, pcDNA3.1 or pcDNA3.1::DnaK. One additional group was immunized by PBS (same quantity, site and time as the DNA injections) and consequently was related as the virulence control group.

\subsection{Antibody response}

\subsubsection{Specificity of anti-DnaK antibodies}

Blood samples were collected from the retro-orbital sinus of non-pregnant mice immunized with PBS, pcDNA3.1 or pcDNA3.1::DnaK for the detection of anti-DnaK-specific antibodies before each DNA injection (days 0, 19, 41), just before the challenge infection (day 61) and at day 68. Blood samples were stored overnight 
at room temperature, centrifuged (600 $\mathrm{g}$, $15 \mathrm{~min}, 4^{\circ} \mathrm{C}$ ) and sera were collected and stored at $-20^{\circ} \mathrm{C}$. The specificity of antiDnaK antibodies was checked by an ELISA test and a western blot.

\section{ELISA}

Recombinant DnaK was used as the antigen and diluted in PBS- $\mathrm{Mg}^{2+}$ free- $\mathrm{Ca}^{2+}$ free at a concentration of $1 \mu \mathrm{g} / \mathrm{mL}$. One hundred microliters were used for coating microculture plates (Nunc-Immuno ${ }^{\mathrm{TM}}$ Plate Maxisorp ${ }^{\mathrm{TM}}$ Surface, Roskilde, Denmark) for $16 \mathrm{~h}$ at $4{ }^{\circ} \mathrm{C}$. Plates were washed 5 times with PBS, $0.05 \%$ Tween 20 . Sera were serially diluted in PBS- $\mathrm{Mg}^{2+}$ free- $\mathrm{Ca}^{2+}$ free, $0.05 \%$ Tween $20: 1 / 200$ for the less reactive serum or starting from $1 / 200$ to $1 / 1600$ for the more reactive serum. Diluted sera $(100 \mu \mathrm{L})$ were incubated for $1 \mathrm{~h}$ at $37^{\circ} \mathrm{C}$, then the plates were washed 5 times with PBS, $0.05 \%$ Tween 20 . Peroxidase-conjugated anti-mouse antibodies $(100 \mu \mathrm{L})$ diluted $1 / 5000$ in $\mathrm{PBS}_{-\mathrm{Mg}^{2+}}$ free-Ca ${ }^{2+}$ free, $0.05 \%$ Tween 20 were incubated for $1 \mathrm{~h}$ at $37^{\circ} \mathrm{C}$, then the plates were washed 5 times with PBS, $0.05 \%$ Tween 20 . Finally, $100 \mu \mathrm{L}$ of ABTS (Chekit ${ }^{\circledR}$ In vitro Diagnosticum, Dr. Bommeli AG, Bern, Switzerland) were used as the substrate. After $20 \mathrm{~min}$ at room temperature, absorbance was read at $414 \mathrm{~nm}$ (ELISA reader). The pooled sera of pcDNA3.1::DnaK immunized mice, collected at day 68 , were used as the reference (pcDNA::DnaK D68) in order to express the results in arbitrary units.

\section{Western blot}

A SDS-polyacrylamide gel (10\%) was performed. After electrophoresis, DnaK recombinant protein $(0.1 \mu \mathrm{g})$ and crude extract of $C$. abortus AB7 were transferred from polyacrylamide gel to a nitrocellulose membrane (Schleicher \& Schuell, PROTRAN®BA83, $0.2 \mu \mathrm{m})$. Subsequently the membrane was blocked in a
1\% milk-PBS Tween 20 buffer at room temperature for $1 \mathrm{~h}$. Immunodetection was performed with $1 / 200$ diluted sera from mice of pcDNA3.1 control and pcDNA3.1::DnaK groups collected at days $0,19,41,61$ and 68 . The lanes were incubated for $1 \mathrm{~h}$ at room temperature. After three washings in PBS- $0.05 \%$ Tween 20, the membrane was incubated for $1 \mathrm{~h}$ with alkaline phosphatase anti-mouse immunoglobulin $\mathrm{G}+\mathrm{M}$ (Caltag, Burlingame, CA, USA) at a dilution of $1 / 2000$ in $1 \%$ milk-PBS. The membrane was washed and alkaline phosphatase activity was detected with NBT-BCIP (Promega) substrate solution.

\subsubsection{Determination of antibody isotypes}

The determination of antibody isotypes was carried out using the ELISA as described above. Briefly, after incubation with dilution of the pooled sera of pcDNA3.1::DnaK vaccinated mice collected at day 61 or day 68 , the isotypes were revealed by adding $100 \mu \mathrm{L}$ of a $1 / 1000$ dilution of peroxidase-conjugated anti-mouse IgG1 or IgG2a or IgG2b (Nordic Immunological Laboratories, Tilburg, The Netherlands). Specific titres for anti-DnaK isotypes were given as the ratio of the absorbance of each IgG subclass/the addition of the absorbance of IgG1, IgG2a and IgG2b.

\subsection{In vitro neutralization assay in the presence of the complement}

The neutralization assay was done by the plaque reduction assay using McCoy cells [27]. Pooled sera (sera of PBS, 1B, pcDNA3.1 or pcDNA3.1::DnaK vaccinated mice) were diluted in $\mathrm{Mg}^{2+}$ free- $\mathrm{Ca}^{2+}$ free phosphate-buffered saline (PBS; $\mathrm{pH} 7.3$ ) containing $10 \%$ fetal bovine serum and $2.5 \%$ guinea pig serum. Two hundred microliters of these dilutions $\left(10^{-1}\right.$ and $\left.10^{-2}\right)$ were added to an equal volume $(200 \mu \mathrm{L})$ of an $10^{4}$ pfu AB7 chlamydial suspension, incubated for $2 \mathrm{~h}$ at 
$37^{\circ} \mathrm{C}$ and then titrated on McCoy cells by the plaque assay method [2]. The results are expressed for each sera by the percent pfu of the PBS control.

\subsection{Chlamydial enumeration in the spleen}

Ten of the 16 non-pregnant immunized mice received an intraperitoneal chlamydial challenge $\left(2 \times 10^{5}\right.$ C. abortus $\left.\mathrm{AB} 7\right)$ at day 68 . Five days after challenge, mice were euthanized because it has been shown that the chlamydial titre in the spleen was maximal at this day [22]. The spleens were aseptically removed, weighed and frozen at $-80^{\circ} \mathrm{C}$. The spleens were individually homogenized in PBS with a glass grinder, diluted in PBS-DEAE (0.01\%) and titrated by plaque assay on McCoy cells, as previously described [23]. The course of infection was evaluated by counting pfu and expressed by $\log _{10}$ pfu.

\subsection{Measurement of DnaK-specific delayed-type hypersensitivity (DTH)}

DTH was evaluated 3 weeks after the third DNA injection on 3 of the 16 nonpregnant immunized mice. The recombinant DnaK protein $(10 \mu \mathrm{g}$ in $30 \mu \mathrm{L})$ was injected into the right hind footpads of three mice, and the same volume of PBS buffer was injected into the left hind footpad as a control. Footpad swelling was measured at 48 and $72 \mathrm{~h}$ after the injection using a dialgauge calliper (Mettallex, Paris, France). The difference of thickness between the right and the left footpads was used as a measure of the DTH response.

\subsection{Statistical tests}

The analysis of the results was performed using the software InStat 2.03 for Macintosh. For all protective results, the mean was calculated using a one way anal- ysis of variance, and a comparison of the means was then carried out through a Student-Newman-Keuls multiple comparison test. The minimal statistical significance was judged at $P<0.05$.

\section{RESULTS}

\subsection{Sequence and expression of the dnaK gene}

Screening of the C. abortus AB7 genetic library with the anti-DnaK monoclonal 18.1 antibody let us identify one clone, labeled $\lambda 70-2$. Restriction endonuclease analysis of the phage arms insert revealed a $13 \mathrm{~kb}$ fragment. After its subcloning in pBluescript ${ }^{\circledR}$ II KS+ and further restriction endonuclease analysis, we obtained a clone labeled pCH704, with a minimal size fragment of $4.3 \mathrm{~kb}$, containing the dnaK gene.

The resulting sequence of the dnaK gene of C. abortus AB7 revealed an open reading frame of $1977 \mathrm{bp}$ encoding a protein of 659 amino acids with a putative calculated molecular weight of $71 \mathrm{kDa}$. The encoded polypeptide was $88 \%$ homologous to DnaK of C. trachomatis and Chlamydophila pneumoniae. The dnaK gene was located at the 3' end of a polycistronic operon comprising two other genes, $h r c A$ and $g r p E$; a similar genetic organization was found in sequenced chlamydial genomes.

The dnaK gene was inserted into the BamHI-XbaI site of the eukaryotic expression vector pcDNA3.1. Production of the $C$. abortus DnaK protein from pcDNA3.1::DnaK construction was confirmed by transfecting the construction into COS-7 cells. The DnaK protein was readily detected using the anti-DnaK monoclonal 18.1 antibody by immunofluorescence in pcDNA3.1::DnaK transfected COS-7 cells (data not shown) and whole cell lysate western blot (Fig. 1) and not in control pcDNA3.1 transfected COS-7 cells. Nevertheless, the molecular mass of 
the recombinant protein $(80 \mathrm{kDa})$ was larger than the molecular mass of purified recombinant DnaK (70 kDa). Since eukaryotic cells expressed the DnaK protein, the pcDNA3.1::DnaK plasmid can consequently be used for DNA immunization.

\subsection{Efficacy of DNA immunization in pregnant mice}

In order to test whether DNA immunization with purified pcDNA3.1::DnaK plasmid generated a protective immunity against $C$. abortus $\mathrm{AB} 7$, immunized mice were mated and challenged with the pathogenic strain C. abortus AB7. The number of live newborn mice in the $1 \mathrm{~B}$ vaccinated group was significantly higher $(P<0.05)$ than in the virulence group and not significantly different from the gestation control group (Fig. 2). The number of live newborn mice in pcDNA3.1 and pcDNA3.1::DnaK vaccinated mice was not significantly different but was significantly lower $(P<0.05)$ than in the $1 \mathrm{~B}$ vaccinated group and significantly higher $(P<0.05)$ than in the virulence group. So, pcDNA3.1 and pcDNA3.1::DnaK vaccinated mice were partially protected from the C. abortus $\mathrm{AB} 7$ challenge.

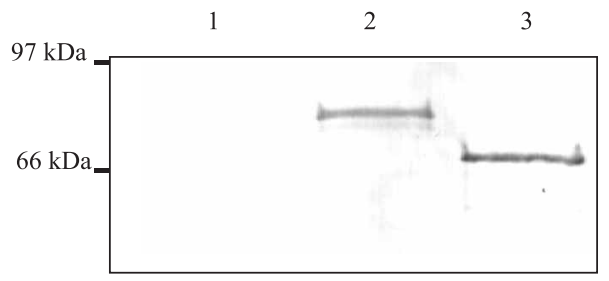

Figure 1. Expression of chlamydial DnaK antigen in eukaryotic transfected cells. The lysates of COS-7 cells transfected with pcDNA3.1 (lane 1) or pcDNA3.1::DnaK (lane 2) plasmids were separated on a SDS-gel electrophoresis and the DnaK protein was detected after a western blot with the anti-DnaK monoclonal 18.1 antibody. The purified recombinant DnaK protein $(0.05 \mu \mathrm{g})$ was used as a control (lane 3$)$. Molecular size is given in the left hand margin.

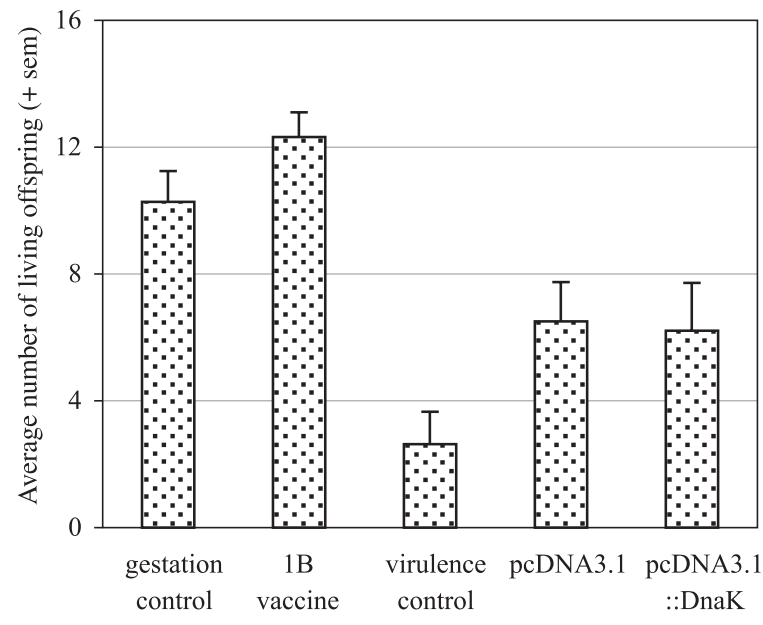

Figure 2. Effect of DNA vaccination on viable offspring. The $1 \mathrm{~B}$ vaccine, the pcDNA3.1 and the pcDNA3.1::DnaK groups were respectively immunized with the live 1B vaccine, the pcDNA3.1 and the pcDNA3.1::DnaK plasmids. The gestation control group was neither infected nor immunized and the virulence group was infected but not immunized. After immunization, the groups were infected after 12 days of gestation. The results were expressed as an average number of living offspring 1 week after birth $(+$ sem). 


\subsection{Anti-DnaK antibody response to DNA vaccination}

An anti-DnaK IgG antibody response was found in the mice immunized with pcDNA3.1::DnaK (Fig. 3). Anti-DnaK IgG were detectable after the first DNA injection, increased after the second injection, reached a maximum after the third pcDNA3.1::DnaK injection and decreased (day 68) after the challenge. This decrease in anti-DnaK IgG antibody titre at day 68 was observed with or without a challenge (data not shown). No DnaK-specific IgG antibodies were identified in the sera from non-immunized mice or from mice immunized with pcDNA3.1.

Then the specificity of the anti-DnaK antibodies were tested by western blot with recombinant purified DnaK (Fig. 4a) and chlamydial extract (Fig. 4b) on the immunized mice sera collected before challenge (day 61) and after challenge (day 68). As expected, no anti-DnaK specific antibodies were detected from sera of mice vaccinated with control plasmid pcDNA3.1 (Fig. 4). Nevertheless, we detected anti-DnaK specific antibodies in sera collected at days 61 and 68 from mice vaccinated with pcDNA3.1::DnaK plasmid. According to the fact that the anti-DnaK monoclonal 18.1 antibody detects a protein with a molecular weight similar to the one detected by the sera of DNA vaccinated mice, we can conclude that the antibodies of these sera are specifically directed against DnaK. Moreover, these sera as well as the monoclonal 18.1 antibody can recognize $\mathrm{DnaK}$ in the $C$. abortus AB7 extract (Fig. 4b).

\subsection{Anti-DnaK antibody isotypes}

As demonstrated in Figure 5, anti-DnaK IgG1 and IgG2a but no IgG2b antibodies were found in the pool of sera of mice immunized with pcDNA3.1::DnaK collected

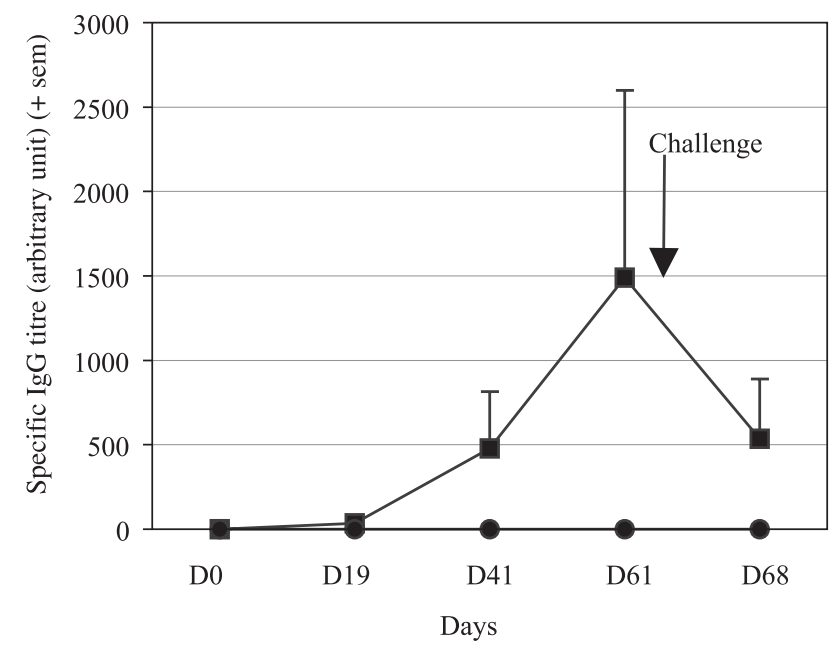

Figure 3. Kinetics of the antibody response of the DNA immunized mice. Mice were intramuscularly immunized with the pcDNA3.1 or the pcDNA3.1::DnaK plasmids at days 0,21 and 42 and challenged at day 63 with the $C$. abortus AB7 bacteria. Sera were collected at different times after the immunization and tested individually by ELISA for the titration of anti-DnaK specific IgG antibodies. The average titre $(+$ sem) of each time had been calculated for pcDNA3.1 $(\bullet)$ or pcDNA3.1::DnaK $(\mathbf{\square})$. 


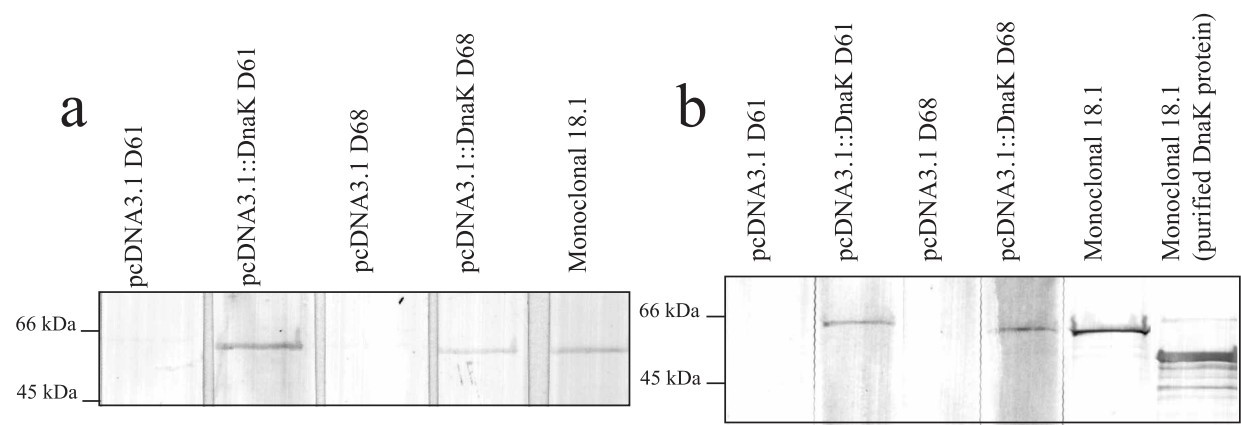

Figure 4. Specificity of the sera of the mice vaccinated with the pcDNA3.1 or the pcDNA3.1::DnaK plasmids analyzed by western blot. Mice were intramuscularly immunized with pcDNA3.1 or pcDNA3.1::DnaK at days 0,21 and 42 and challenged at day 63 with C. abortus AB7. The sera collected at days 61 and 68 were tested by western blot using the purified recombinant DnaK protein $\left(0.1 \mu \mathrm{g} /\right.$ lane) (3a) or C. abortus AB7 extracts $\left(8 \times 10^{6}\right.$ bacteria/lane) $(3 \mathrm{~b})$ as antigens. Molecular size is given in the left-hand margin.

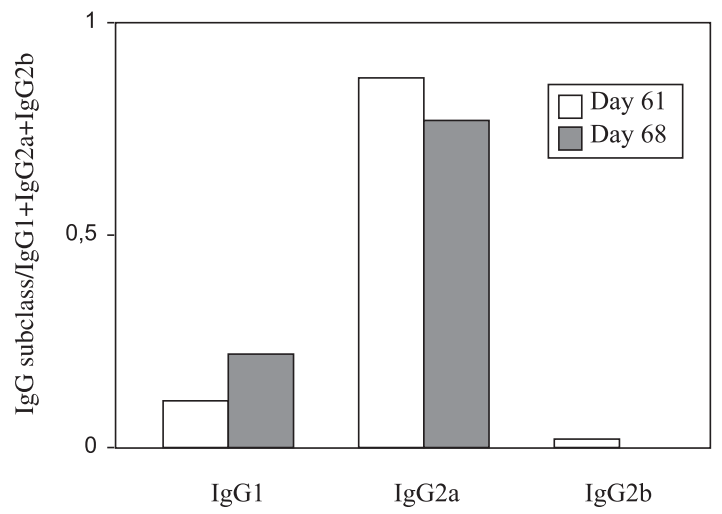

Figure 5. Determination of the anti-DnaK IgG subclasses in the pooled sera of mice immunized with pcDNA3.1::DnaK. ELISA assayed the sera for the isotype of anti-DnaK specific antibodies. The IgG subclasses were investigated at day 61, three weeks after the third DNA injection and at day 68, 5 days after the challenge. The absorbance of each IgG subclass was divided by the value obtained by the addition of the absorbance of each IgG subclass.

at days 61 and 68. IgG2a levels were respectively 7.9 and 3.4 times higher than IgG1 levels in the sera collected at days 61 and 68. These data indicate that plasmid vaccination using the dnaK gene both generates $\mathrm{IgG} 1$ and $\mathrm{IgG} 2 \mathrm{a}$ antibodies with a higher proportion of IgG2a.

\subsection{The neutralization effect of anti-DnaK antibodies in the presence of a complement}

The $10^{-1}$ and $10^{-2}$ dilution of $1 \mathrm{~B}$ vaccinated mice sera reduced the infectivity of $C$. abortus (90 and 68\% respectively) (Fig. 6). 


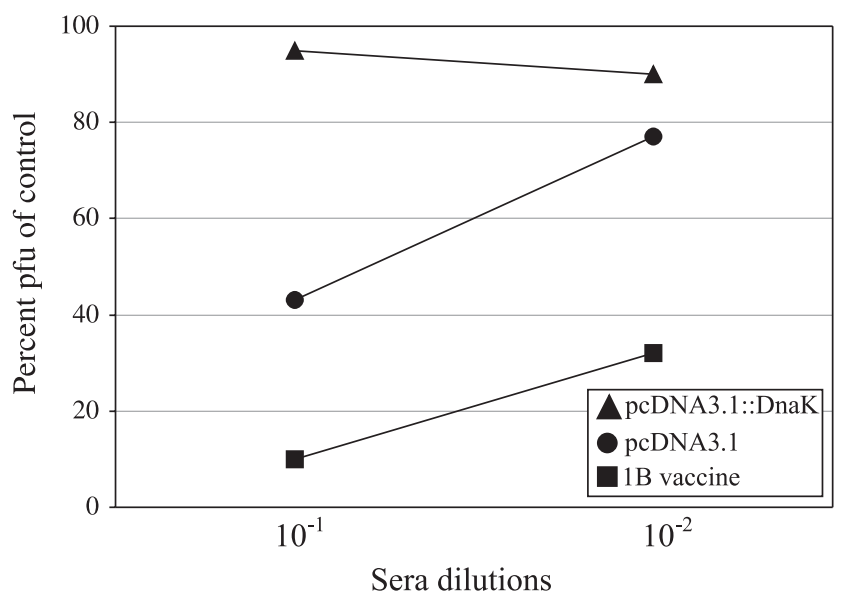

Figure 6. Neutralizing effect of the $1 \mathrm{~B}$, the pcDNA3.1 or the pcDNA3.1::DnaK vaccinated mice sera. The mice sera collected at day 61 were $10^{-1}$ and $10^{-2}$ diluted and then added to the chlamydial suspension. These solutions had been titrated and the results were expressed in percent pfu of control.

There was no significant difference between the effect of $10^{-2}$ dilution of pcDNA3.1 and pcDNA3.1::DnaK vaccinated mice sera since they did not reduce the infectivity of the bacteria. Nevertheless, the $10^{-1}$ dilution of the pcDNA3.1 vaccinated mice sera reduced in vitro $(57 \%)$ the infectivity of C. abortus while the $10^{-1}$ dilution of pcDNA3.1::DnaK vaccinated mice sera did not. The pcDNA3.1::DnaK vaccinated mice sera failed to reduce the pfu number induced by C. abortus.

\subsection{Clearance of $C$. abortus $\mathrm{AB} 7$ challenge in the spleen of non-pregnant mice}

Chlamydial titres in the spleens of $1 \mathrm{~B}$ vaccinated mice were significantly lower $(p<0.05)$ than the ones observed in the virulence group. Thus, 1B vaccinated mice were partially protected against chlamydial infection (Fig. 7). Chlamydial titres of pcDNA3.1 and pcDNA3.1::DnaK vaccinated mice spleens did not show significant difference with the virulence group. Thus,
DNA vaccination induced no splenic protection in non-pregnant mice.

\subsection{DTH response}

No positive DTH response was detected at $48 \mathrm{~h}$ in 3 mice (out of 3 ) immunized with pcDNA3.1::DnaK. On the contrary, 2 (out of 3) $1 \mathrm{~B}$ immunized mice showed a DTH response (data not shown). No difference was found between the DTH responses elicited with non vaccinated or pcDNA3.1 vaccinated mice. For these two groups, only one mouse (out of 3 ) was slightly positive.

\section{DISCUSSION}

DNA vaccination elicits both humoral and cellular immune responses specifically against the protective protein of interest [13]. DNA vaccines can be considered as marker vaccines because they allow the serological differentiation between vaccinated and naturally infected animals. They could therefore compensate for the major 


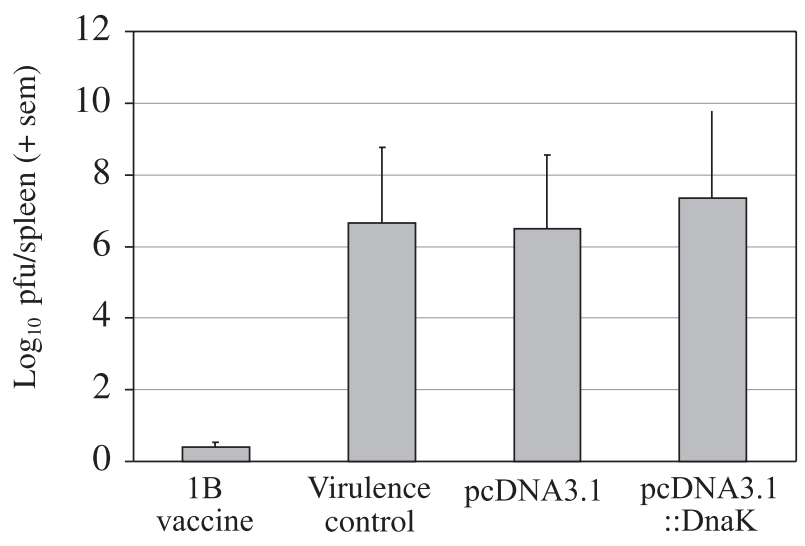

Figure 7. Chlamydial clearance in spleen of the non immunized or the immunized mice. Mice were immunized by three intramuscular injections of the pcDNA3.1 or the pcDNA3.1::DnaK plasmids or one injection of the 1B vaccine. Then they were challenged with C. abortus AB7 and euthanized 5 days after the challenge. The quantities of bacteria in spleens are expressed in $\log _{10}$ pfu per spleen $(+$ sem).

drawback of the actual anti-C. abortus commercialized vaccine namely the $1 \mathrm{~B}$ temperature-sensitive mutant [22] which induces the same immune response as the virulent $C$. abortus strains. Moreover, DNA immunization appears to be a promising method to resolve $C$. abortus infections since DNA vaccines have already been used with more or less success in Chlamydia trachomatis [33], C. pneumoniae [19] and in Chlamydophila psittaci [32] vaccination models.

Different antigens could be used for the DNA vaccination against Chlamydiaceae. In this study, we evaluated the protective immunity induced by the $d n a K$ gene of $C$. abortus. Indeed, the bacterial heat shock proteins and more particularly, the DnaK protein (Hsp70) of C. abortus are known to be strong immunogens $[1,34]$. DnaK is located in the outer membrane of $C$. trachomatis and is accessible to the monoclonal antibody [21]. Moreover, a DnaK encoding DNA vaccine has already been successfully used in a tuberculosis model [16]. Hsp have potential immunopathological properties [34] but in our study, no adverse effect was observed after DNA immunization with pcDNA3.1::DnaK.

The dnaK gene of C. abortus AB7 was isolated from a genomic library after screening with an anti-DnaK monoclonal antibody. The corresponding DnaK protein of $C$. abortus $\mathrm{AB} 7$ revealed high homology (88\%) with DnaK of C. trachomatis and $C$. pneumoniae. This confirms the extreme inter-species conservation of heat shock proteins throughout evolution. The dnaK gene was inserted in a eukaryotic vector and its expression was confirmed in transiently transfected COS-7 cells. Subsequently, the vaccine construct was used to immunize mice.

Intramuscular DNA immunization gave a partial protection against intraperitoneal challenge as shown by the reduction of the average number of living offspring in the dnaK immunized mice. Mice immunized with the vaccinal $1 \mathrm{~B}$ strain were fully protected having the same average number of living offspring as mice belonging to the gestation control group. However, immunization with the dnaK gene gave the same 
reduction in living offspring as immunization with the control vector. Thus, dnaK vaccination of pregnant mice probably non-specifically protected the mice from abortion and could not consequently be allocated to an anti-DnaK specific immune response. Identical non-specific immune responses elicited by DNA vaccination have been discussed by other authors [15, 26] and can possibly be explained by immunostimulating properties of $\mathrm{CpG}$ motifs which are present in bacterial DNA [31]. Indeed, bacterial DNA containing unmethylated CpG motifs appear to be recognized as a foreign body in vertebrates and stimulate a wide array of immune cells and notably increase the Th1 immune response in mice [8]. The CpG motif has even been proposed as a potential adjuvant [10].

In the present study, the non-specific immune response induced by DNA immunization failed to protect the non-pregnant mice since no reduction of the mean pfu number in the spleens of DNA immunized mice was observed. On the contrary, significant bacterial clearance was observed in the spleens of $1 \mathrm{~B}$ immunized mice. Since the chlamydial clearance in the spleen is known to be principally due to the cytotoxic T cells [6], we hypothesized that the dnaK immunization elicited an insufficient or a non-protective cellular immune response. This was confirmed since no DTH reaction was found in dnaK immunized mice. Therefore, DNA immunization with the dnaK gene of $C$. abortus is not able to elicit a protective cellular immune response.

Nevertheless, we demonstrated a DnaKspecific humoral response with predominant IgG2a antibodies suggesting a Th1 type immune response. The antibodies of dnaK immunized mice sera were able to recognize the DnaK recombinant protein as well as the native chlamydial DnaK protein. Moreover, the isotype of the protective antibodies was known to be $\operatorname{IgG} 2 \mathrm{a}$ in $C$. abortus infection [11]. However, despite their specificity and their isotypes, these
anti-DnaK antibodies had no neutralizing effect on the chlamydial penetration in McCoy cells. Surprisingly, the sera of mice immunized with the plasmid control showed a neutralizing effect. At present, we have not explained this phenomenon but further investigations will be performed.

The production of DnaK detected in transiently transfected COS-7 cells and the anti-DnaK humoral immune response induced in vaccinated mice argue for an in vivo expression. However, the quantity of recombinant antigen produced in vivo following intramuscular DNA vaccination is usually weak [20] and in the present study could not elicit a protective immune response. In order to enhance the immune responses induced by dnaK DNA immunization, it would be interesting to combine a final proteic boost to the DNA immunization since it was admitted that this combined strategy increases both humoral and cellular immune response in comparison with protein or DNA vaccination alone [30].

In conclusion, we demonstrated that the immunization with the dnaK gene of $C$. abortus AB7 induced a weak humoral immune response unable to mediate a specific protection in pregnant and non-pregnant mice. Before we conclude that the DnaK protein is not a protective antigen, it would be necessary to assess if the enhancement of the immune response level influences the protective effect of the DnaK DNA vaccination.

\section{ACKNOWLEDGMENTS}

The authors thank Laurence HibrandSaint Oyant for helpful discussion and critical readings of this manuscript and Françoise Bernard for excellent technical assistance. Céline Héchard was supported by a grant from INRA-Région Centre. 


\section{REFERENCES}

[1] Anderson I.E., Craggs J.K., Dunbar S., Herring A.J., Cloning and expression of the $75 \mathrm{kDa}$ DnaK-like protein of Chlamydia psittaci and the evaluation of the recombinant protein by immunoblotting and indirect ELISA, Vet. Microbiol. 58 (1997) 295-307.

[2] Banks J., Eddie B., Schachter J., Meyer K.F., Plaque formation by Chlamydia in L cells, Infect. Immun. 1 (1970) 259-262.

[3] Birkelund S., Larsen B., Holm A., Lundemose A.G., Christiansen G., Characterization of a linear epitope on Chlamydia trachomatis serovar L2 DnaK-like protein, Infect. Immun. 62 (1994) 2051-2057.

[4] Birkelund S., Mygind P., Holm A., Larsen B., Beck F., Christiansen G., Characterization of two conformational epitopes of the Chlamydia trachomatis serovar L2 DnaK immunogen, Infect. Immun. 64 (1996) 810-817.

[5] Buxton D., Potential danger to pregnant women of Chlamydia psittaci from sheep, Vet. Rec. 118 (1986) 510-511.

[6] Buzoni-Gatel D., Guilloteau L., Bernard F., Bernard S., Chardes T., Rocca A., Protection against Chlamydia psittaci in mice conferred by Lyt-2+ T cells, Immunology 77 (1992) 284-288.

[7] Caldwell H.D., Kromhout J., Schachter J., Purification and partial characterization of the major outer membrane protein of Chlamydia trachomatis, Infect. Immun. 31 (1981) 11611176.

[8] Chu R.S., Targoni O.S., Krieg A.M., Lehmann P.V., Harding C.V., CpG oligodeoxynucleotides act as adjuvants that switch on T helper 1 (Th1) immunity, J. Exp. Med. 186 (1997) 1623-1631.

[9] Davis H.L., Whalen R.G., Demeneix B.A., Direct gene transfer into skeletal muscle in vivo: factors affecting efficiency of transfer and stability of expression, Hum. Gene Ther. 4 (1993) 151-159.

[10] Davis H.L., Weeratna R., Waldschmidt T.J., Tygrett L., Schorr J., Krieg A.M., Weeranta R., CpG DNA is a potent enhancer of specific immunity in mice immunized with recombinant hepatitis B surface antigen, J. Immunol. 160 (1998) 870-876.

[11] De Sa C., Souriau A., Bernard F., Salinas J., Rodolakis A., An oligomer of the major outer membrane protein of Chlamydia psittaci is recognized by monoclonal antibodies which protect mice from abortion, Infect. Immun. 63 (1995) 4912-4916.

[12] Denamur E., Sayada C., Souriau A., Orfila J., Rodolakis A., Elion J., Restriction pattern of the major outer-membrane protein gene provides evidence for a homogeneous invasive group among ruminant isolates of Chlamydia psittaci, J. Gen. Microbiol. 137 (1991) 2525-2530.

[13] Donnelly J.J., Ulmer J.B., Shiver J.W., Liu M.A., DNA vaccines, Annu. Rev. Immunol. 15 (1997) 617-648.

[14] Faye P., Charton A., Mage C., Bernard C., Le Layec C., Propriétés hémagglutinantes du «virus » de l'avortement enzootique des petits ruminants (souches de « Rakeia » d'origine ovine et caprine), Bull. Acad. Vet. Fr. 45 (1972) 169-173.

[15] Krieg A.M., Love-Homan L., Yi A.K., Harty J.T., CpG DNA induces sustained IL-12 expression in vivo and resistance to Listeria monocytogenes challenge, J. Immunol. 161 (1998) 24282434.

[16] Lowrie D.B., Silva C.L., Colston M.J., Ragno S., Tascon R.E., Protection against tuberculosis by a plasmid DNA vaccine, Vaccine 15 (1997) 834-838.

[17] Lundemose A.G., Birkelund S., Larsen P.M., Fey S.J., Christiansen G., Characterization and identification of early proteins in Chlamydia trachomatis serovar L2 by two-dimensional gel electrophoresis, Infect. Immun. 58 (1990) 2478-2486.

[18] Papp J.R., Shewen P.E., Localization of chronic Chlamydia psittaci infection in the reproductive tract of sheep, J. Infect. Dis. 174 (1996) 1296-1302.

[19] Penttila T., Vuola J.M., Puurula V., Anttila M., Sarvas M., Rautonen N., Makela P.H., Puolakkainen M., Immunity to Chlamydia pneumoniae induced by vaccination with DNA vectors expressing a cytoplasmic protein (Hsp60) or outer membrane proteins (MOMP and Omp2), Vaccine 19 (2000) 1256-1265.

[20] Prud'homme G.J., Lawson B.R., Chang Y., Theofilopoulos A.N., Immunotherapeutic gene transfer into muscle, Trends Immunol. 22 (2001) 149-155.

[21] Raulston J.E., Paul T.R., Knight S.T., Wyrick P.B., Localization of Chlamydia trachomatis heat shock proteins 60 and 70 during infection of a human endometrial epithelial cell line in vitro, Infect. Immun. 66 (1998) 2323-2329.

[22] Rodolakis A., In vitro and in vivo properties of chemically induced temperature-sensitive mutants of Chlamydia psittaci var. ovis: screening in a murine model, Infect. Immun. 42 (1983) 525-530.

[23] Rodolakis A., Chancerelle L., [Plaque assay for Chlamydia psittaci in tissue samples (in French)], Ann. Microbiol. (Inst. Pasteur) B 128 (1977) 81-85.

[24] Rodolakis A., Souriau A., Response of ewes to temperature-sensitive mutants of Chlamydia psittaci (var ovis) obtained by NTG mutagenesis, Ann. Rech. Vet. 14 (1983) 155-161.

[25] Rodolakis A., Souriau A., Response of goats to vaccination with temperature-sensitive mutants 
of Chlamydia psittaci obtained by nitrosoguanidine mutagenesis, Am. J. Vet. Res. 47 (1986) 2627-2631.

[26] Sato Y., Roman M., Tighe H., Lee D., Corr M., Nguyen M.D., Silverman G.J., Lotz M., Carson D.A., Raz E., Immunostimulatory DNA sequences necessary for effective intradermal gene immunization, Science 273 (1996) 352-354.

[27] Schachter J., Banks J., Sugg N., Sung M., Storz J., Meyer K.F., Serotyping of Chlamydia. I. Isolates of ovine origin, Infect. Immun. 9 (1974) 92-94.

[28] Schild H., Arnold-Schild D., Lammert E., Rammensee H.G., Stress proteins and immunity mediated by cytotoxic T lymphocytes, Curr. Opin. Immunol. 11 (1999) 109-113.

[29] Strugnell R.A., Drew D., Mercieca J., DiNatale S., Firez N., Dunstan S.J., Simmons C.P., Vadolas J., DNA vaccines for bacterial infections, Immunol. Cell Biol. 75 (1997) 364-369.

[30] Tanghe A., D’Souza S., Rosseels V., Denis O., Ottenhoff T.H., Dalemans W., Wheeler C., Huygen K., Improved immunogenicity and pro- tective efficacy of a tuberculosis DNA vaccine encoding $\mathrm{Ag} 85$ by protein boosting, Infect. Immun. 69 (2001) 3041-3047.

[31] Tighe H., Corr M., Roman M., Raz E., Gene vaccination: plasmid DNA is more than just a blueprint, Immunol. Today 19 (1998) 89-97.

[32] Vanrompay D., Cox E., Volckaert G., Goddeeris B., Turkeys are protected from infection with Chlamydia psittaci by plasmid DNA vaccination against the major outer membrane protein, Clin. Exp. Immunol. 118 (1999) 49-55.

[33] Zhang D., Yang X., Berry J., Shen C., McClarty G., Brunham R.C., DNA vaccination with the major outer-membrane protein gene induces acquired immunity to Chlamydia trachomatis (mouse pneumonitis) infection, J. Infect. Dis. 176 (1997) 1035-1040.

[34] Zugel U., Kaufmann S.H., Role of heat shock proteins in protection from and pathogenesis of infectious diseases, Clin. Microbiol. Rev. 12 (1999) 19-39. 